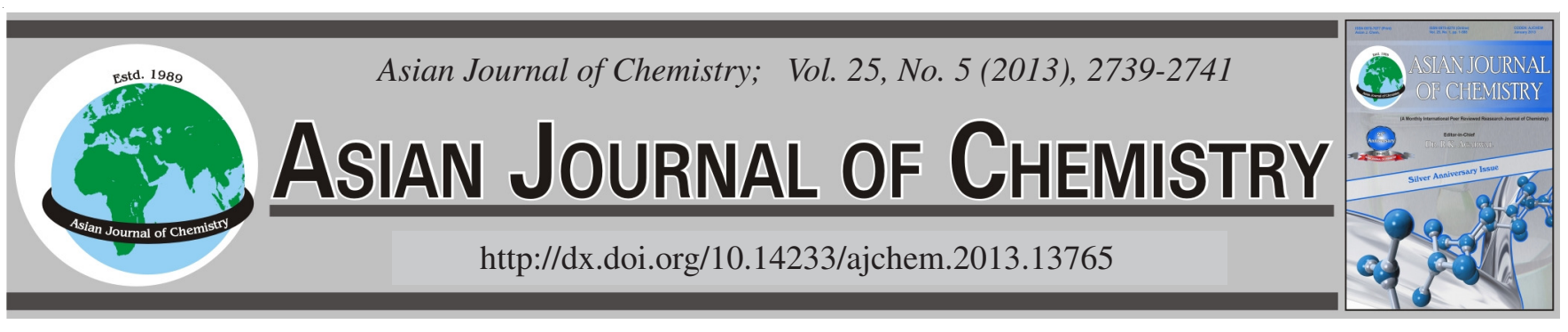

\title{
Dyeing Behaviour of $\gamma$-Irradiated Cotton Using Amaltas (Cassia fistula) Bark Extracts
}

\author{
Shahid Adeel ${ }^{1, *}$, FaZAl-UR-Rehman $^{2, *}$, Tahsin Gulzar $^{2}$, IJaz Ahmad Bhatti $^{3}$, Summia QAiser $^{2}$ and Anam Abid ${ }^{1}$
}

${ }^{1}$ Department of Chemistry, Government College University, Faisalabad-38000, Pakistan
${ }^{2}$ Department of Applied Chemistry, Government College University, Faisalabad-38000, Pakistan
${ }^{3}$ Department of Chemistry \& Biochemistry, University of Agriculture, Faisalabad-38040, Pakistan

*Corresponding authors: E-mail: shahidadeelchemist@gmail.com; furminhas@gmail.com

\begin{abstract}
Amaltas bark (Cassia fistula) has been used as natural colourant for dyeing of un-irradiated and irradiated cotton fabric. Both cotton fabric and dye powder has been exposed to absorbed doses of 2, 4, 6, 8 and $10 \mathrm{kGy}$ using Cs $137 \gamma$-irradiator. Dyeing parameters such as temperature and time were optimized. To improve colour strength pre and post mordanting using copper and iron as mordants was carried out. Suggested ISO standard methods for colour, fastness to light, washing and rubbing were employed to observe fastness properties. It is observed that dyeing of irradiated fabric at $50^{\circ} \mathrm{C}$ for $50 \mathrm{~min}$ using $\mathrm{Cu}$ as pre-mordant $(4 \%)$ has not only given good colour strength with darker shades but also acceptable fastness properties are obtained. It is concluded that $\gamma$-irradiation has a promising effect in improvement of colour strength and colourfastness properties and it can be applied on other fabrics dyed with other natural colourants.
\end{abstract}

Key Words: Cotton, Colourfastness properties, Dyeing, Spectraflash SF-600.

\section{INTRODUCTION}

Recently, most of the commercial dyers and textiles export houses have started re-looking to the maximum possibilities of using natural dyes for dyeing and printing of different textiles for targeting niche market. It is only because natural dyes produce uncommon, soothing and soft shades as compared to synthetic dyes. While synthetic dyes which are carcinogenic and have health hazards by polluted water and other harmfulness to human body by producing toxic chemicals during their synthesis and finishing process ${ }^{1}$. Being fast, having eco-friendly nature and easily biodegradable and its extraction and application under mild conditions, natural dyes are gaining worldwide attraction due to awareness of people toward environment. Hence there is a revival of natural dyes in the world of textiles ${ }^{2}$. Cotton is considered as the most plentiful and renewable biopolymer in nature. It is widely used in textile industry due to its cheap and linear nature. It contains three-hydroxyl group in which primary hydroxyl group is responsible for bonding with dye molecule. Different techniques have been carried out to modify the surface of the cellulose fibers. Among them UV and $\gamma$-irradiation has promising effect. Previously in our work, we have found that $\gamma$-irradiation improves the shrinking and wrinkling resistance of fibers, rise dye uptake rate, improves fixation as well shade of dye. It adds value in colouration and does not change morphology of dye as well as physical structure of cotton ${ }^{3}$.
Keeping in view such useful effects of $\gamma$-irradiation, the present study has been carried out using natural colourants extracted from Amaltas (Cassia fistulas) bark. The present study is a part of practical applicability of Amaltas (Cassia fistula) bark as natural dye onto irradiated fabric. It is highly effective laxative, antioxidant, hypoglycemic, antiviral, liver protector, pain reliever and acts as fever reducing agent. It contains tannins, phlobaphenes and some oxyanthraquinone substances. Stem bark contains lupeol, $\beta$-sitosterol, etc. 1,8dihydroxy-6-methoxy-3-methyl anthraquinone is the main colourant found in stem bark of Amaltas.

The aim of present study is to improve the colour strength of irradiated cotton as well as to enhance colourfastness properties of irradiated fabric using extracts obtained from Amaltas (Cassia fistula) bark.

\section{EXPERIMENTAL}

Fresh and dried barks of Cassia fistula (commonly known as Amaltas) were selected as source of natural colourant, which was obtained from University of Agriculture, Faisalabad, Pakistan. Plain woven, bleached and mercerized cotton was obtained from Noor Fatima Fabrics Mills (Faisalabad, Pakistan). Barks of Amaltas were chopped into small pieces, ground finely using a food blender and sieved through sieve of 20 meshes to obtain homogenous powder of uniform particle 
size. The powder was then stored in opaque, airtight plastic jars for further experiments. All the chemicals used for extraction, dyeing and mordanting were of commercial grades.

Irradiation and extraction process: Both cotton and bark powder were exposed to absorbed doses of 2, 4, 6, 8 and 10 kGy using Cs-137 source at Nuclear Institute of Agriculture and Biology (NIAB), Faisalabad Pakistan ${ }^{4}$. For extraction of dye, one part of un-irradiated (NRP) and irradiated powder (RP) was boiled with thirty parts of DDW [M:L; 1:30] for $1 \mathrm{~h}$. The obtained extract was used to dye un-irradiated (NRC) and irradiated cotton fabrics (RC). To obtain extraction and dyeing condition for further experiments all dyed (irradiated and unirradiated) fabrics were sent to CIE Lab system for evaluation of colour strength in order to observe the effect of $\gamma$-radiation on extraction.

Dyeing and mordanting process: Dyeing parameters such as temperature and time were optimized using optimum conditions of extraction. To observe the effect of temperature dyeing were carried out for irradiated cotton using extracts of un-irradiated powder at $40,50,60,70$ and $80^{\circ} \mathrm{C}$. In another experiments, dyeing was carried out for 30, 40, 50, 60 and 70 min using un-irradiated and irradiated cotton. Dyed samples were extensively washed with cold and hot water to remove any unfixed dyed material and finally dried at ambient temperature. To improve colour strength of dye on irradiated cotton, pre and post mordanting using copper and iron as mordants was carried out. For that purpose, 2, 4, 6, 8 and $10 \%$ solution of copper and iron were used ${ }^{5}$.

Colour measurement and fastness properties: The colour strength values were obtained in CIE Lab system using spectraflash SF 600 with illuminant D65 having $10^{\circ}$ observer. Suggested standard methods of ISO for colourfastness such as colour fastness to light (ISO $105 \mathrm{BO} 2$ ), colourfastness to washing (ISO $105 \mathrm{CO} 3$ ) and colourfastness to rubbing (ISO $105 \times 12$ ) were employed to study the effect of $\gamma$-irradiation on colourfastness properties of cotton using extracts of unirradiated Amaltas bark powder ${ }^{6}$.

\section{RESULTS AND DISCUSSION}

The results displayed in Fig. 1 show that $\gamma$-ray treatment of cotton has a remarkable effect on its dyeing with Amaltas bark extract. It is found that at low dose, the cotton fabric did not activate enough to uptake more dye, due to which low colour strength is observed. At high dose, the fabric becomes enough photomodified and insoluble impurities along with colourant get maximum chance to sorb due to which low strength with uneven shades are observed. Some loss in mass of fabric might be occurred as well degradation of fabric may be possible due to which less colour strength is observed ${ }^{7}$. At $6 \mathrm{kGy}$, the surface of fabric become photomodified and the surface of cellulose may convert into carboxylic acid, which upon dyeing form firm bond with dye molecules, which upon investigation in spectraflash give high colour strength. The influence of $\gamma$-irradiation on extraction did not give such significant results. So it is concluded that $\gamma$-ray treatment of cotton at optimum does ( $6 \mathrm{kGy})$ has raise the dye uptake ability of cotton but also added more value in colouration using unirradiated dye powder.

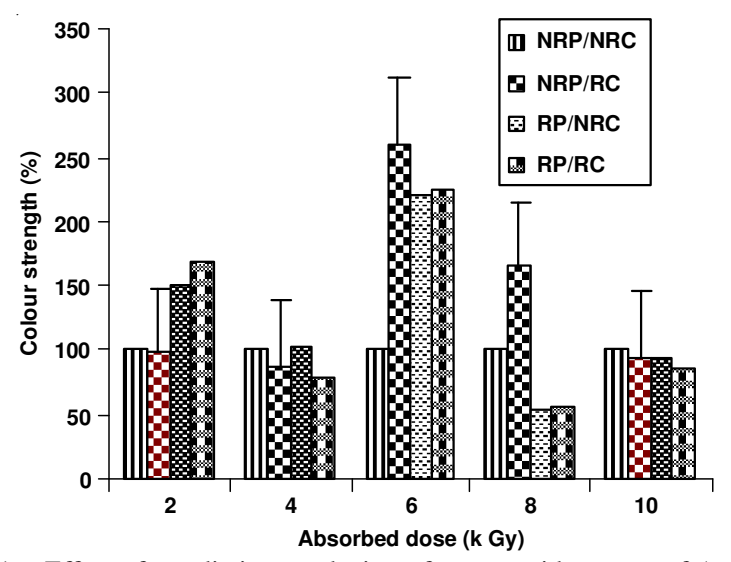

Fig. 1. Effect of $\gamma$-radiation on dyeing of cotton with extract of Amaltas bark powder

Temperature plays an important role in dyeing because the rate of dyeing and the rate of striping depend upon it. In general, acceptable results can be obtained at moderate temperatures, e.g. between $40-60{ }^{\circ} \mathrm{C}^{8}$. Previously it is found that at low temperature dye molecules in aggregates along with impurities rush onto fabric and cause unevenness. While at very high temperature, the colourant may face hydrolytic degradation and the impurities get significant chance to sorb onto photomodied fabrics, which give low colour strength upon investigation in spectraflash. The effect of temperature on the dye ability of the irradiated cotton fabrics was conducted at different temperatures $\left(40-80^{\circ} \mathrm{C}\right)$ given in Fig. 2 . using extracts of un-irradiated powder. The colour strength values show that upon gradual raising the temperature more dye molecules are attached but at high temperatures dye components probably start to decompose resulting in uneven dyeing. At $50{ }^{\circ} \mathrm{C}$ the affinity of dye molecules to the cellulose fibers was maximum and even dyeing was observed. So optimized dyeing temperature is $50^{\circ} \mathrm{C}$.

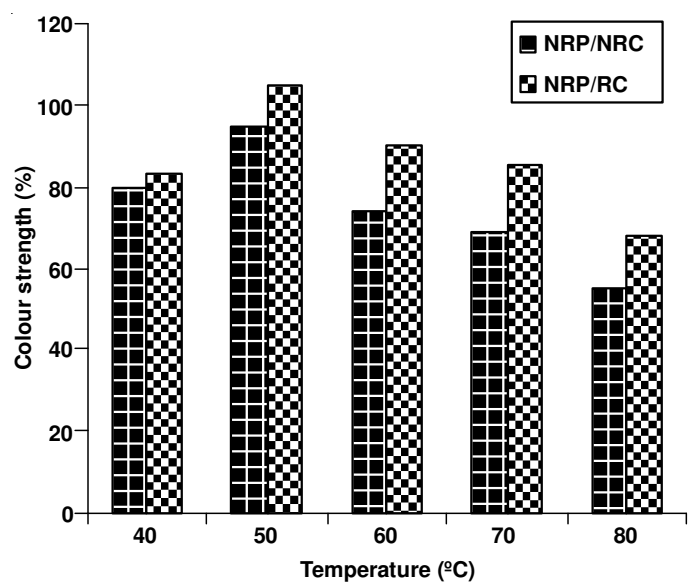

Fig. 2. Effect of dyeing temperature on dyeing of irradiated cotton (6 kGy) using extract of un-irradiated Amaltas bark powder

In dyeing process time is very important because during constant heat for a long-time, decomposition of the dye material might occur while short-time causes incomplete sorption of colourant onto irradiated fabric. The colour strength values displayed in Fig. 3 demonstrate these effects. The samples obtained were either uneven or incompletely dyed while dyeing for short time. The optimized dyeing time is $50 \mathrm{~min}$ when the 


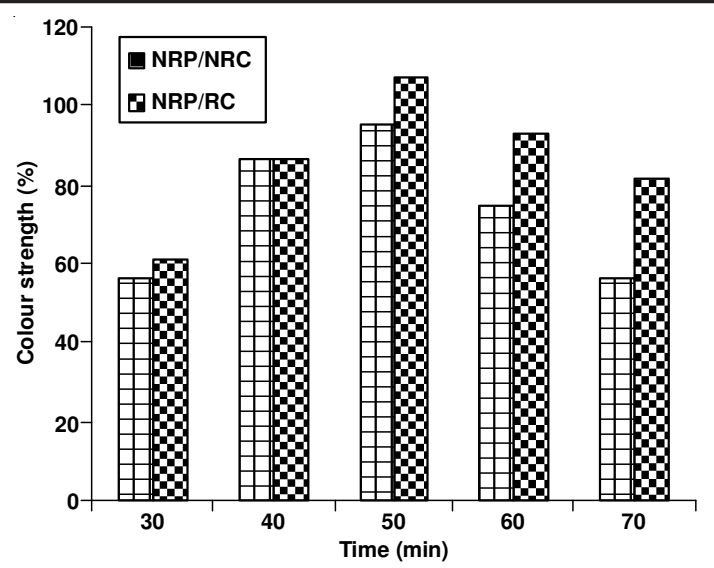

Fig. 3. Effect of dyeing time on dyeing of irradiated cotton (6 kGy) usin $g$ extract of un-irradiated Amaltas bark powder

sample is evenly and completely dyed as the result of the maximal dye-uptake of the cotton fibers. The decline in the dyeability after $50 \mathrm{~min}$. may be attributed to the desorption of the dye molecules as a consequence of long dyeing time.

The role of mordanting is very vital in natural dyeing process. As natural colourants are less substantive with fibers, so they need some metals salt for their fixation, improvement of colour strength as well as colour fastness properties. The results shown in Fig. 4 for pre-mordanting and Fig. 5 for post mordanting show that mordanting with $6 \%$ solution of copper gave good colour strength and acceptable fastness properties onto irradiated fabric. The low colour strength of iron mordanting is due to the fact that iron having low reduction power does not significantly interact with dye material due to which dye aggregates gather onto modified fabric and causes unevenness with dull shades giving low strength in spectra flash. Hence the optimum pre and post mordanting condition is $6 \%$ copper as mordant.

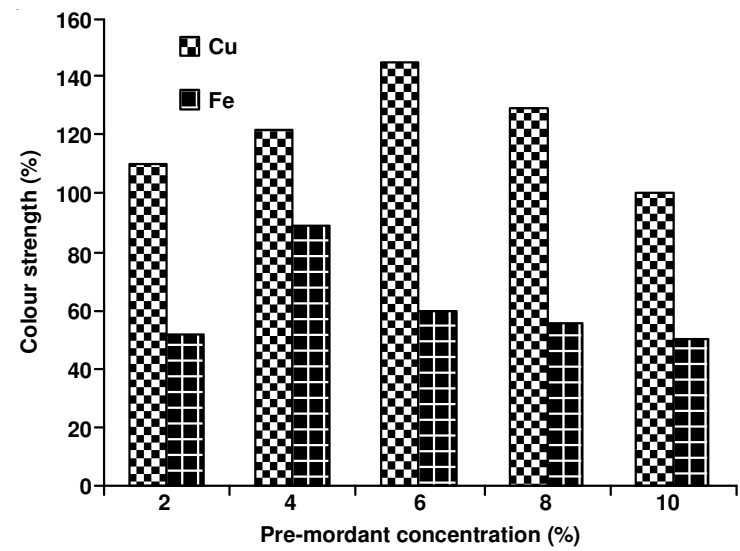

Fig. 4. Effect of pre-mordanting on dyeing of irradiated cotton (6 kGy) using extract of un-irradiated Amaltas bark powder

Table-1 shows that the irradiated fabric dyed with the extract of un-irradiated bark of Amaltas (Cassia fistula) powder exhibits good fastness properties. The superior washing property of the colourant is due to the kinetics and thermodynamic effects of the metal complex formation in aqueous medium. The good colour fastness properties might be attributed to benzene ring and conjugated system present in dye which make firm bonding with modified fabric and upon exposure to agencies such as

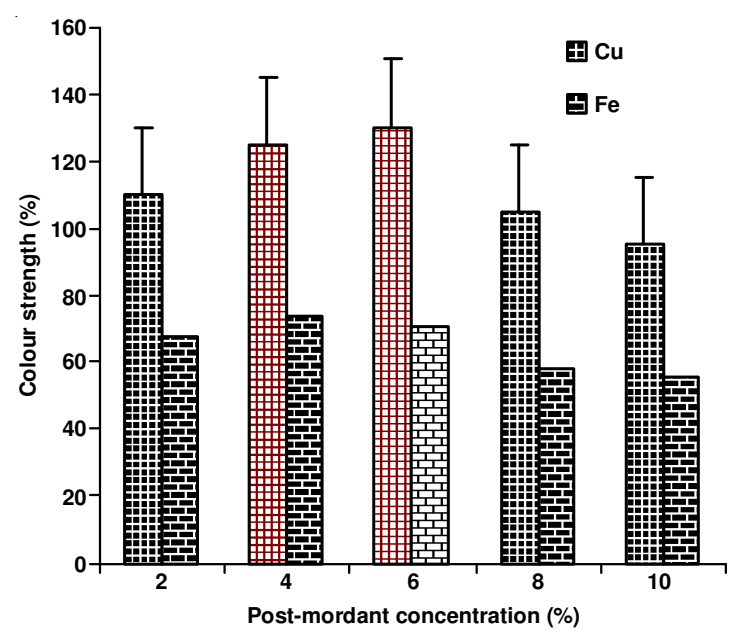

Fig. 5. Effect of post-mordanting on dyeing of irradiated cotton (6 kGy) using extract of un-irradiated Amaltas bark powder

\section{TABLE-1}

EFFECT of $\gamma$-RADIATION ON COLOURFASTNESS PROPERTIES OF IRRADIATED COTTON (RC, 6 kGy) DYED WITH EXTRACT OF UN-IRRADIATED AMALTAS BARK POWDER

\begin{tabular}{lcccc}
\hline Optimum conditions & $\begin{array}{c}\text { Light } \\
\text { fastness }\end{array}$ & $\begin{array}{c}\text { Washing } \\
\text { fastness }\end{array}$ & $\begin{array}{c}\text { Dry } \\
\text { rubbing } \\
\text { fastness }\end{array}$ & $\begin{array}{c}\text { Wet } \\
\text { rubbing } \\
\text { fastness }\end{array}$ \\
\hline Absorbed dose $(\mathrm{RC}, 6 \mathrm{kGy})$ & 4 & $4-5$ & $4-5$ & $4-5$ \\
Temperature $\left(50^{\circ} \mathrm{C}\right)$ & $3-4$ & 4 & $4-5$ & 4 \\
Time $(50 \min )$ & 4 & $3-4$ & $4-5$ & 4 \\
Pre-Mordant $(\mathrm{Cu}=6 \%)$ & 4 & 4 & $4-5$ & $4-5$ \\
\hline
\end{tabular}

heat, detergent, light and rubbing show resistance ${ }^{9}$. Hence $\gamma$ irradiation of fabric has not only enhanced these properties but also improved the colour strength.

\section{Conclusion}

The natural dye extracted from Amaltas bark powder might be used as a possible substitute of synthetic dyes having banned aryl-amine moieties. Amaltas bark powder being good laxative is one of dyes that provide a source of natural colourant for dyeing of cotton by using high energy radiation. Extraction of dye from un-irradiated powder and its application onto irradiated fabric give acceptable fastness properties. It is found that such radiations are useful in application of natural colourants from other plants using different fabrics under the influence of radiations such as UV, microwave, ultrasonic and $\gamma$-irradiation.

\section{REFERENCES}

1. M. Muneer, I.A. Bhatti and S. Adeel, Asian J. Chem., 22, 7453 (2010).

2. S. Adeel, S. Ali, I.A. Bhatti and F. Zsila, Asian J. Chem., 21, 3493 (2009).

3. I.A. Bhatti, S. Adeel, R. Nadeem and T. Asghar, Radiat. Phys. Chem., 81, 264 (2012).

4. I.A. Bhatti, S. Adeel, M.A. Jamal, M. Safdar and M. Abbas, Radiat. Phys. Chem., 79, 622 (2010).

5. S. Adeel, I.A. Bhatti, A. Kausar and E. Osman, Indian J. Fib. Text Res., 37, 87 (2012).

6. S. Naz, I.A. Bhatti and S. Adeel, Indian J. Fib. Text Res., 36, 132 (2010).

7. E. Takacs, L. Wojnarovits, C.S. Foldvary, J. Borsa and I. Sajo, Radiat. Phys. Chem., 27, 1837 (2000).

8. A.A. Ansari and B.D. Thakur, Colourage, 47, 15 (2000).

9. V.A. Popoola, J. Appl. Polym. Sci., 77, 752 (2000). 